\title{
Au cœur de la création artistique : le combat de Jacob avec l'Ange
}

Je crois avoir lu Edipe sur la route en apnée, dans un état de bouleversement intense qui est resté imprimé en moi et se réactive dès que j'en reprends la lecture. Il fait partie de ces livres rares qui comptent dans une vie et occupent durablement le champ intérieur.

Pour qui interroge les processus de création et s'efforce d'en capter le caractère singulier, initiatique, aux antipodes du divertissement dans lequel l'art se voit de plus en plus relégué, l'œuvre ${ }^{18}$ d'Henry Bauchau offre un témoignage irremplaçable. Y sont rapportés les moments les plus intenses de la création, non pas comme vérités abstraites mais comme expériences spirituelles ancrées dans l'existence la plus incarnée. Ce qui impressionne chez Bauchau, c'est l'éclatante présence de l'art dans toutes ses dimensions, sans prétention métaphysique ni, à l'opposé, banalisation des pratiques, mais avant tout comme expérience humaine et source irremplaçable de connaissance. C'est une pensée du processus qui se déploie là, soulignant les transformations constantes qui s'opèrent dans une vie plutôt que les projets esthétiques eux-mêmes. À travers l'art sous toutes ses formes, musique, danse, peinture, sculpture, poésie, c'est avant tout à un processus d'autopoïèse que l'on assiste, auto-poïèse de l'écrivain, auto-poïèse des personnages. L'œuvre en soi n'est jamais fétichisée. Elle n'apparaît même jamais comme objectif, mais seulement comme trace, éphémère la plupart du temps, d'une métamorphose, preuve d'un moment d'existence et d'un palier atteint au cours d'un processus d'individuation. "C'est le trajet de la flèche qui compte et non la cible", enseigne l'art zen du tir à l'arc. Il est en effet moins question chez Bauchau de l'autonomie de l'art telle qu'elle s'est peu à peu construite en Occident que de l'émergence de l'œuvre dans l'intense présent de sa naissance. Pourtant l'intime compréhension qu'a l'écrivain de l'expérience artistique lui permet d'envisager l'art dans la diversité de ses fonctions, de l'auto-poìèse à la constitution du groupe social, de l'aventure individuelle la plus solitaire au rituel communautaire, du plus spirituel au plus pulsionnel, de la matière la plus irréductible à l'immatérialité la plus totale, du simple partage à la pratique la plus élitiste.

La plupart des grandes questions relatives à l'art sont posées au cours du roman, toujours reliées à l'expérience existentielle, évitant ainsi les pièges

18 Bien qu'Edipe sur la route soit l'objet principal de cette étude, nous l'avons élargie à tout le cycle œdipien: Antigone, Diotime et les lions, Les Vallées du bonheur profond... 
de la théorisation. En une époque où les attitudes commerciales et communicationnelles instrumentalisent l'art et entretiennent une confusion délétère à son endroit, la méditation de Bauchau sur l'existence humaine dans sa complexité enracine l'art dans sa nécessité même.

Mais pourquoi recourir à l'image du "Combat de Jacob avec l'Ange" pour accompagner cette réflexion sur la création artistique? Une conception romantique égarée dans une époque rebelle au pathos se manifesterait-elle ainsi, selon laquelle la douleur de l'artiste aux prises avec ses combats intérieurs prendrait le pas sur l'artisanat et l'essence collective de l'œuvre souvent préférés par la modernité? Il y aurait beaucoup à dire sur ce sujet, car le soubassement expérientiel de l'art par delà le XIX ${ }^{\mathrm{e}}$ siècle n'a sans doute pas encore été exploré à sa juste mesure et le rôle de la souffrance humaine dans la création contemporaine trop rapidement relativisé par les courants de pensée actuels. Parmi ceux-ci, l'anthropologie et les Cultural studies ont certes fort utilement contribué à questionner la domination esthétique occidentale, de même que des analyses de plus en plus nombreuses mettent à juste titre en cause certains dogmatismes de la psychanalyse. Il n'empêche que l'Occident a développé au cours des derniers siècles d'individualisme philosophique un rapport singulier à la création: la domination de l'écrit et du rationalisme a entraîné de profondes répercussions - fût-ce parfois par rébellion - sur la conception de l'art et de l'artiste, les modes d'apprentissage et la réception esthétique. Cela même caractérise la culture européenne et mériterait l'intérêt d'une anthropologie culturelle, telle que l'appelait de ses voux Hannah Arendt ${ }^{19}$. On comprendrait sans doute mieux pourquoi, bien au-delà du romantisme, la création artistique demeure un processus douloureux et un itinéraire spirituel incompatible avec le divertissement.

Toutefois - et pour l'instant, je m'en tiendrai là - la métaphore de Jacob s'est simplement imposée à moi alors que je recommandais avec chaleur la lecture d'Edipe à des amis. Je me suis en effet surprise à leur décrire spontanément sous cette image biblique l'épisode de la Vague, puis celui du temple rouge de Clios au commencement d'Antigone. Pourtant, oserais-je l'avouer, je ne me souvenais plus alors précisément du texte biblique et l'allusion soufflée par mon inconscient se confondait alors dans mon esprit avec les multiples combats archétypaux qui mettent les héros grecs aux prises avec des monstres. Je ne m'attarderais pas sur cette fantaisie de mon imagination si je n'avais eu la surprise, en ouvrant le Journal d'CEdipe sur la route, de lire à la première page: "Passant devant St Sulpice, j'entre pour aller voir La Lutte de Jacob avec l'ange de Delacroix. Touché comme toujours par la beauté, la gravité de la scène. L'homme précipite toutes ses forces dans la lutte, l'ange se contente de le contenir sans effort apparent,

19 Hannah Arendt, Condition de l'homme moderne, Paris, Calmann-Levy, 1961 et 1983. 
avec un magnifique geste de retenue. Je me suis assis dans la mauvaise lumière qui règne là et j'ai laissé l'œuvre me pénétrer, m'entourer, non sans une certaine tristesse. Le temps n'est plus pour moi de me lancer dans la lutte. Je n'ai pas non plus la grâce, la puissante réserve de l'ange. Il faut que je trouve ma place dans cet affrontement décisif, mais mon esprit ignore et mon corps ne sent pas encore où elle se trouve. ${ }^{20}$ Bauchau reviendra à plusieurs reprises dans son Journal sur cette scène qui l'accompagnera pendant toute la gestation du roman et ce dernier donnera la preuve éclatante que le temps de lutter était encore actuel : à 70 ans, le poète prouvera que le moment était bien venu d'engager avec l'Ange le combat décisif qui conduirait un CEdipe pacifié aux portes du bois sacré...

Pourtant, était-ce la scène de l'Ancien Testament ou la toile de Delacroix qui habitait l'écrivain? Laquelle de ces images s'était infiltrée dans son imaginaire pour donner vie à CEdipe? Les deux représentations ne s'étaientelles pas plutôt entremêlées pour conférer au héros son envergure exceptionnelle? D'un côté, la puissante portée symbolique de l'épisode biblique qui décrit la transformation spirituelle d'un homme ordinaire - l'homme vil, marqué dans sa chair par sa lutte intérieure, devient patriarche, transmetteur de valeurs spirituelles et fondateur d'une lignée. De l'autre, l'accent mis sur la violence incontrôlée de Jacob contre la transcendance.

Il était donc nécessaire dans un premier temps de relire le texte biblique ${ }^{\mathbf{2 1}}$ : Jacob, personnage assez peu recommandable, après avoir trompé son père Isaac, extorqué sa bénédiction, usurpé le droit d'aînesse d'Esaü son frère jumeau, fui au loin, trompé son beau-père Laban, revient sur le conseil de Dieu dans son pays avec femmes et enfants. Esaü l'attend avec une armée de 400 hommes, bien décidé à en découdre avec ce frère félon. La veille de la rencontre, Jacob effrayé reste seul et la Bible dit sans plus d'effet d'annonce: "C'est alors qu'un homme lutta avec lui jusqu'au lever de l'aurore". Après lui avoir déboîté la hanche, l'homme sans nom demande à partir, bénit Jacob qui l'en prie et, attestant sa victoire à l'étonnement du lecteur, le baptise d'un nouveau nom, Israël, lui promettant une nombreuse descendance. La métamorphose de Jacob est accomplie.

Comment avais-je bien pu, dans mon inconscient, assimiler l'énigmatique épisode biblique sans nul doute bien mal connu de moi-même, à un combat héroïque contre un monstre mythique? Pourquoi avais-je retenu de l'expression "le combat de Jacob contre l'ange", l'idée erronée d'une lutte titanesque à l'image de celles d'Hercule ou de Thésée - la mémoire de ce dernier irriguant il est vrai de nombreuses ouvres de Bauchau? Ces

20 Henry Bauchau, Jour après jour. Journal d'CEdipe sur la route (1983-1989), Arles, Actes Sud, 2003, p. 11.

21 Genèse, 32. 
questions ne cessaient de me hanter. Il n'y avait pourtant aucune trace de violences héroïques dans le texte biblique, aucune figure céleste manifeste, aucune blessure spectaculaire. Seuls un texte elliptique, un "homme" mystérieux et une boiterie indélébile. En revanche, chez Delacroix, la violence furieuse d'un Jacob sauvage, vêtu d'une peau de bête, se déchaîne contre un ange aux ailes ostensiblement déployées, puissant, calme et protecteur. Qu'avais-je donc intuitivement perçu chez CEdipe qu'avait bien dû déposer Bauchau dans son livre, pour que j'y associe de moi-même, quoique à mon insu, la référence à Jacob?

Que soient présents dans le roman les symboles de l'homme aux prises avec son hybris et sa violence destructrice, c'est incontestable: Clios et sa rage dévastatrice, l'aventure du labyrinthe et le meurtre du Minotaure, le meurtre de Laïos, l'épisode souterrain du peuple des Hautes collines, la lutte fratricide d'Étéocle et Polynice... Sans cesse les héros se heurtent aux pulsions obscures qui les animent et les détruisent. L'œuvre de Bauchau est toujours placée sous le signe du chaos et du combat. Mais la plupart du temps, comme dans les mythologies païennes, le héros affronte une image apparemment étrangère à lui-même qui reflète son versant ombreux. Or dans l'histoire de Jacob, c'est l'inverse. Là en effet, ce n'est pas le monstre qui apparaît en miroir, mais l'ange, la part de lumière. Le guerrier, voire la Bête, c'est bien Jacob lui-même, tandis que l'adversaire incarne son versant lumineux. Toute l'histoire d'Edipe sur la route consiste en une progressive initiation de personnages dévastés par les combats incessants qui ont émaillé leur vie et la culpabilité qui les dévore. Ils devront apprendre à ne plus refuser leur part lumineuse et à s'autoriser le pardon. Dans les mythes grecs, c'est la bête qu'il faut terrasser pour accéder à l'humanité, dans la Bible, c'est l'ange qu'il faut accueillir pour renouer avec Dieu après la faute originelle. Or si, pour décrire le roman à mes amis, je n'ai intuitivement pas recouru à la mythologie grecque pourtant propice à rendre compte du domptage par l'humain de sa part instinctuelle, c'est qu'il y avait chez Bauchau un besoin d'une autre sorte, une aspiration qui devait impérativement trouver une autre forme. L'aspiration à desserrer l'étau infernal de la culpabilité semble avoir renvoyé l'écrivain à ses racines judéo-chrétiennes caractérisées par le péché originel de l'Ancien Testament et le pardon par l'amour du christianisme. Ainsi n'est-ce pas tant le monstre qui restait à vaincre que l'indulgence envers soi-même qu'il fallait découvrir. En infligeant une "blessure de lumière "22 à la hanche de Jacob, l'ange le marque du sceau divin et, comme dans "l'échange de sang" réclamé par Antigone pour la fresque de $\mathrm{Clios}^{23}$, il enclenche la transformation psychique. Tout au

22 "Clios pense que ce n'est plus un puits mais une blessure de lumière", Henry Bauchau, Antigone, Arles, Actes Sud, 1997, p. 15.

23 Antigone est déçue de la fresque de Clios et lui révèle ce qui manque dans le combat de Thésée 
long de l'itinéraire initiatique, Antigone incarne l'amour inconditionnel qui éclaire ses deux compagnons à chaque palier de leur douloureux processus d'individuation.

Ainsi, bien loin d'être le héros auquel renvoie naturellement la mythologie grecque, l'CEdipe de Bauchau apparaît avant tout comme un héros chrétien.

\section{Art et processus d'individuation}

Observons comment, en tant que voies de sagesse, l'activité puis la création artistiques conduisent progressivement CEdipe et Clios vers la libération - ou l'"éveil", pour emprunter un terme bouddhiste. Pour le psychanalyste Bauchau qui se sent paria, exilé de sa propre cité par le soupçon des hommes ${ }^{24}$, l'art se montre en effet capable d'épauler l'introspection et de favoriser la guérison.

Edipe sur la route commence au moment où, terrassé par la culpabilité, CEdipe s'arrache à l'enceinte carcérale de son moi barricadé, à la protection factice des remparts de Thèbes qu'il avait dans sa vanité fait construire autrefois, pour se jeter en mendiant sur les routes, affrontant tragiquement l'inconnu' ${ }^{25}$. Il faut enfin que l'insupportable cesse! Le héros se lance alors sans aucune compassion pour lui-même dans sa terrible introspection. La cruelle question de la liberté est posée d'emblée lorsque Clios lui assène la question vertigineuse: "Est-ce que tu étais libre lorsque tu as tué ton père et épousé ta mère? "26 Pour les deux hommes, il va dès lors s'agir, à travers d'innombrables épreuves souvent métaphorisées par la création artistique, de se délivrer des illusions de la toute-puissance pour découvrir, à travers l'acceptation de contraintes extrêmes, où les mène leur véritable liberté.

Observons comment l'art accompagne chaque étape de leur itinéraire spirituel.

1- Au commencement du roman, l'existence de l'art dans leur vie ne se pose même pas. Une totale privation sensorielle marque le premier chapitre,

contre le Minotaure, le sang que les combattants doivent s'échanger pour que l'initiation soit accomplie. Ibid, p. 13.

24 Il est fait allusion ici au soupçon qui pesa sur l'écrivain après la guerre, du fait de son engagement dans les mouvements intellectuels catholiques belges de rénovation sociale et de certaine amitié encombrante avec un sympathisant nazi. On lira sur ce sujet Geneviève Duchenne, Vincent Dujardin, Myriam Watthee-Delmotte, Henry Bauchau dans la tourmente du XX siècle. Configurations historiques et imaginaires, Bruxelles, Le Cri, 2008.

25 Le Journal d'Edipe sur la route s'ouvre avec l'épigraphe de saint Jean de la Croix: "Pour aller où tu ne sais pas/Va par où tu ne sais pas".

26 Henry Bauchau, Edipe sur la route, Arles, Actes Sud, 1990, p. 37. 
le rendant quasiment intolérable, avec ses phrases brèves et l'omniscience insupportable d'un présent vécu par le lecteur comme intrusif: le silence règne - CEdipe ne parle plus; la sécheresse des yeux, du coeur et de la terre ainsi que la brûlure du soleil imposent leur loi; les corps sont pesants et raidis par la fatigue et le malheur, le regard, annihilé. La compression intérieure est telle qu'elle déclenche par réflexe de survie le commencement de l'errance et de l'itinéraire initiatique.

2- Un premier palier est atteint à l'arrivée chez Diotime, la guérisseuse, double imaginaire de Blanche Jouve, la psychanalyste qui libéra le désir d'écrire chez l'écrivain. Les femmes chantent et lidée d'art comme une sorte d'évocation fantomatique, fait son apparition: grâce à la paix et la bienveillance de Diotime, Antigone se ressouvient des temps heureux où CEdipe chantait pour ses enfants. En révélant à Antigone qu'CEdipe appartient à une lignée de Clairchantants, la guérisseuse fait savoir que ce don doit être partagé et ne peut en aucun cas être gardé pour soi. Le don musical et poétique porte en lui-même l'obligation du lien social. À partir de cette révélation, CEdipe ne peut plus se dérober aux autres. Ainsi, par l'intermédiaire d'Antigone, Diotime libère la mémoire affective d'CEdipe et ouvre la digue expressive et sensorielle. Dès lors et très lentement, Edipe réapprend à vivre en retrouvant l'usage de ses sens atrophiés par la douleur: Antigone lui fait toucher le tissu du manteau bleu offert par Diotime, Clios lui en décrit la couleur, un bleu grave qui pénètre l'âme de sa douceur profonde, Antigone lui révèle à son tour sa filiation paternelle de Clairchantant et parvient par la musique à lever le tabou qu'est encore l'évocation du père assassiné. C'est d'ailleurs à ces heureuses réminiscences sensorielles que l'on doit le premier lâcher prise d'CEdipe qui sort enfin de son mutisme pour sa première confession.

Peu après, au cours d'un émouvant transfert poétique, Clios quant à lui reconstruit patiemment la perception émoussée de son compagnon en partageant avec lui ses propres sensations de l'espace traversé ensemble. C'est le paysage façonné par les sens de Clios qui pénètre CEdipe par l'oreille et lui redonne peu à peu l'usage harmonisé de ses propres sens. Lart en sa matérialité et non en sa seule idéalité prépare son entrée.

Pour l'heure, bénéficiant lui aussi de la libération de la parole, Clios se met à raconter son histoire et, sur l'évocation amoureuse d'Alcyon, le souvenir émerveillé de la danse et de la musique envahissent et revivifient tout le champ affectif. Deux expériences d'art s'entremêlent: d'un côté, l'art apollinien d'Alcyon, nimbé d'immatérialité et de transparence, animé de souffle pur et de la spiritualité qui émane du chant des voyelles, et de l'autre, l'art dionysiaque, pulsionnel de Clios, incarné par le clan de la danse et nourri tant par la vitalité et la joie de l'espace que par la pesanteur et le 
chaos dévastateur. Malgré l'intensité de leur échange amoureux, il manque à Alcyon et à son art le goût de l'incarnation et c'est sans doute ce que symbolise sa mort: l'esprit ne peut se déployer dans la séparation de l'âme et du corps.

3- En ranimant sa propre mémoire jusque-là anesthésiée, Clios ravive en CEdipe et en lui-même la nécessité des pratiques artistiques ensevelies: la flûte et la danse apparaissent donc et raniment la vie qui stagnait, enfermée dans la souffrance de chacun. Il ne s'agit encore que d'une expression rude et barbare qui cherche l'expression plus que la forme, mais grâce à elle, le souffle vital et le corps se remettent à fonctionner.

Après le souffle et le corps, c'est une autre matière - la matière du monde - qui les sollicite: ils se mettent à pétrir la terre, sculpter le minéral et le végétal, y lisant des signes énigmatiques mais annonciateurs de changements vitaux (le sourire de la pierre, la source qui murmure dans une branche d'olivier...). La danse de transe se met quant à elle à charrier pêlemêle le souvenir du clan, le reliement à la communauté humaine, à la figure mythique de la Mère, à la mémoire ancestrale...

Plus que d'art, il ne s'agit encore que de pratiques spontanées, nécessaires à la renaissance sensorielle et psychique, proches de l'art-thérapie, mais elles s'avèrent irremplaçables en cette étape cruciale où s'amorcent les changements psychiques fondamentaux.

4- Survient alors le bouleversant épisode de la Vague où une étape capitale est franchie, celle de l'auto-poïèse accomplie au miroir de la poïèse. Ce n'est certes encore que l'homunculus ${ }^{27}$ goethéen qui se laisse entrevoir à travers sa bulle de cristal, un être encore inabouti, mais le processus de transmutation est en marche. Les trois héros vont pour combattre leurs folies respectives s'attaquer à la sculpture de la falaise, à l'assaut du matériau qu'en véritable artiste, Edipe a désigné comme le seul qui puisse contenir sa vérité. Pour la première fois, l'intensité de l'action créative les libère de leur souffrance tenace et les mène jusqu'à l'œuvre. C'est alors le sommet - le climax de l'œuvre -, le "Combat de Jacob avec l'ange". Au lieu de se tourner contre lui-même comme il l'avait fait jusque-là, CEdipe parvient à extérioriser sa souffrance et la projette sauvagement sur la pierre intraitable. Cette colère, Bauchau écrivain la connaît bien. Il l'a reconnue chez MichelAnge qui, à un ami effrayé de le voir s'épuiser à travailler le marbre avec tant de rage, s'écriait: "Comment ne serais-je pas furieux contre ce marbre qui m'empêche de voir la statue qu'il emprisonne encore? "28 Le futur CEdipe - l'CEdipe à naître - doit absolument s'échapper de sa forteresse invivable

27 Allusion au second Faust de Goethe.

28 Henry Bauchau, L'Écriture à l'écoute, Arles, Actes Sud, 2000, p. 147. 
pour trouver dans une matière étrangère à lui-même le remède à son mal. Or cet Autre, incarné par la falaise rouge qui détient la solution de son énigme, il doit l'interroger sans répit, se mettre à son écoute pour découvrir ce qu'elle lui cache encore. Le corps à corps sauvage destiné à arracher son secret à la pierre le meurtrit, les meurtrit puisque CEdipe entraîne ses deux compagnons dans sa folie créatrice, extirpant une à une les images salvatrices qu'elle recèle. Devant les exigences de l'art, il est contraint, pour imprimer sa quête dans la roche et en faire jaillir la scène libératrice, d'inventer de nouvelles techniques (fabriquer les outils adéquats, se suspendre au-dessus du vide au bout d'une corde, par exemple), les seules aptes à faire surgir du matériau "trouvé-créé" ${ }^{29}$, la réponse qui lui est propre. Aucune technique partagée et déjà éprouvée ne saurait lui ouvrir une voie qui ne peut être que singulière. À travers les gestes dictés par sa seule expérience dans l'acuité de sa douleur impartageable, il doit trouver son propre cheminement expressif. "Penser ou faire ne suffit. Surgir" écrit-il dans ses Aphorismes. Il hurle donc, vomit, frappe la roche et finit par faire naitre dans sa fureur créatrice la barque qui permettra aux trois rameurs et à leur guide aveugle de franchir la vague déferlante. La forme - l'ange - se révèle dans la falaise et au lieu des monstres qui peuplent habituellement son esprit, CEdipe y rencontre enfin le guide calme et assuré, la bonne image fondatrice, promesse de salut. CEdipe-Jacob a reconnu l'Ange en lui-même et se trouve enfin prêt à transmettre son humanité désormais éclairée à sa descendance...

À travers une telle quête désespérée, n'est-ce pas le processus de création lui-même qui est décrit là? L'invention d'un matériau arraché par l'humain à la matière ${ }^{30}$ et marqué par son aspiration spirituelle, la résistance énigmatique de ce matériau ${ }^{31}$ qui impose sa loi et oblige l'artiste à conquérir de haute lutte la forme (le sens?), l'invention de techniques imposées par la nécessité intérieure, l'action totalement incarnée de l'artiste qui engage tout son être dans la spiritualisation de la matière rebelle, l'ignorance dans laquelle se trouve le créateur de ce qu'il cherche véritablement et que le temps du travail dévoile pas à pas, l'enjeu vital de l'acte créateur, l'exultation devant l'œuvre enfin achevée, le deuil qui s'ensuit devant l'inévitable

\footnotetext{
29 Allusion au concept-clé chez Winnicott d' "objet transitionnel", celui qui est à la fois trouvé parmi les objets du monde et investi d'une valeur inégalable par l'enfant. C'est lui qui permet à l'enfant, puis, sous d'autres formes à l'adulte, de surmonter l'expérience de solitude, de séparation originelle et d'établir une communication vitale entre le dedans de l'intériorité et le dehors du monde partagé. Cf. Donald W. Winnicott, Jeu et réalité. L'espace potentiel, Paris, Gallimard, 1975.

30 Adorno écrit des pages magnifiques dans sa Théorie esthétique sur ce concept de matériau caractérisé par les efforts des hommes pour spiritualiser la matière à l'aide d'inventions techniques. La technique est précisément ce qui, au fil de l'histoire, a permis au génie humain de transformer la matière en y déposant son désir de transformation spirituelle.

31 Un concept qu'Adorno encore théorisera dans ses écrits esthétiques, en écho à l'expérience de bien des artistes.
} 
effacement par le temps des larmes versées, la reconnaissance émue des hommes que l'œuvre illumine et... la nécessité de reprendre la route.

En tout cas, c'est bien ce premier acte créateur partagé avec ses compagnons de souffrance qui amène CEdipe vers son propre pardon. Levant enfin la terrible sanction qu'il avait prononcée contre lui-même, il revient parmi les hommes.

5- À partir de là, il est prêt à donner aux autres. Reconnu comme l'incarnation de la souffrance humaine, il est consacré aède par la communauté et accepte la charge qu'on lui confie d'entretenir la mémoire collective. Toutefois, si la musique comme la danse sont les facteurs de lien dont la communauté a besoin pour cultiver sa mémoire et inventer son futur, elles ne sont pas immunisées contre les dangers qui guettent la société des hommes: il est donc indispensable que l'art cultive la forme pour ne pas abandonner le champ expressif au cri qui menace d'ameuter la horde sauvage. L'art est lui aussi hélas capable d'outrepasser ses limites et de plonger les hommes dans "la lutte du sang et de l'esprit"32. L'effrayante scène au cours de laquelle Edipe aboie sa douleur à la lune en donne un témoignage saisissant. Au nom de sa souffrance, l'artiste ne peut se dédouaner de sa responsabilité collective. Celle-ci l'engage irrémédiablement.

6- Dès lors, le roman ne cesse de rappeler l'essence éphémère de l'art et sa vocation d'accompagner les constantes transformations de l'être ou du groupe social. L’ouvre ne peut être fétichisée ni réifiée car elle est matière vivante. Elle émane de la "circonstance", ce moment fortuit et impermanent qui a surgi un jour au sein de l'expérience humaine mais qui peu à peu s'y est réenfoui. C'est pourquoi les statuettes d'CEdipe sont irrémédiablement vouées à l'abandon, une à une, sur la route, avant l'arrivée à Colone...

Et en effet, le processus de l'art serait incomplet s'il ne devait aboutir qu'au sauvetage de l'individu. Sa dimension spirituelle provient de l'étape ultime du processus créateur: la dissolution de l'individu dans "une instance plus vaste que celle qui a vécu l'expérience „33, celle qui conduit Bauchau, à la fin de son livre L'Écriture à l'écoute, à délaisser le je au profit du on. C'est à partir de là seulement que l'œuvre quitte son créateur pour s'ouvrir à l'humanité et la nourrir de ce qu'elle a découvert au cours de son élaboration. L'ouvre devient art à partir du moment où elle se détache de l'expérience individuelle pour parler de condition humaine et ouvrir un accès partagé à la connaissance. C'est ici qu'entre en jeu dans l'œuvre de Bauchau, à la fin d'Antigone, une des plus belles pages qui soient. Antigone, murée dans une grotte sur ordre de Créon, est en train de mourir. Elle parle sa mort - la

32 Henry Bauchau, Edipe sur la route, op. cit., p. 188.

33 Henry Bauchau, L'Écriture à l'écoute, op. cit., p. 151. 
mort qu'elle est en train de vivre - la disparition de l'air, ses dernières sensations, ses dernières méditations, ses derniers souvenirs, ses dernières hallucinations... Et le miracle advient alors que, par le pouvoir mystérieux de cet énigmatique temps présent propre à l'écriture poétique de Bauchau, le lecteur se sent lui-même peu à peu absorbé par le processus de dissolution qui emporte Antigone : le je et le $t u$ commencent à se mêler dans la délibération intérieure de l'héroïne sacrifiée, faisant place à une présence qui ne cesse de croître, une autre Antigone, celle qui, par la musique d'Io, est en train de devenir insensiblement un modèle pour les générations qui s'annoncent. Par le chant inspiré d'Io, Antigone passe insensiblement le flambeau à la vivante, à celle qui sera capable de transmettre aux hommes son expérience ultime. L'art se révèle seul capable de transmettre l'expérience profonde pourtant réputée intransmissible, à ouvrir l'accès à la connaissance et à transcender le temps.

"Quand je chante, Clios, je suis Antigone. Je chante ce que sa vie me dicte, mais je dois rester Io. Regarde, la petite pleure parce qu'elle m'a senti trop loin d'elle et trop longtemps. L'autre qui m'écoutait avec tant d'attention commence à s'inquiéter aussi. Il faut que je m'occupe d'eux, Antigone a décidé d'habiter ce lieu avec nous, elle ne va pas disparaître."

Non, je ne vais pas disparaître pendant qu'Io prend sa fille dans ses bras et sourit à son fils. Ils sont bientôt rassurés pourtant je vois, ou je devine, que sous le sourire d'Io coulent mes larmes. Celles que je ne pouvais m'empêcher de verser pendant que je lançais à Créon ce cri que j'ai oublié, ce cri sorti de moi sans que je le sache, qui en lui disant non, disait oui à l'espoir, minime, altéré, qui était, qui est, qui sera indéfiniment.

[...] Io est l'Antigone du futur, bien plus intrépide, plus lucide que je n'étais. Je vois qu'elle a peur comme moi, qu'elle ne le cache pas et je lui sais gré de me montrer à tous comme je suis, un peu égarée, vite effrayée et pourtant capable, je ne sais comment, de répondre à ce qu'exige cette voix qui parfois m'habite et dont celle d'Io est l'incomparable écho. Voix faite pour parler au cour de tous et traverser le temps. Pourquoi traverser le temps? Est-ce que je ne puis pas m'effacer comme les autres? Dans le chant d'Io on entend qu'il n'y a rien d'illimité que le présent. C'est ce que j'ai appris sur la route, ce que K. a trouvé dans la musique et qu'CEdipe a fini par découvrir à Colone. ${ }^{34}$

34 Henry Bauchau, Antigone, op. cit., pp. 349-350. 


\section{Une méditation sur l'art}

À travers l'itinéraire initiatique d'CEdipe et de ses compagnons ${ }^{35}$, Bauchau offre une méditation sur l'art dont on aimerait synthétiser ici les grandes orientations.

Il faut tout d'abord le rappeler: pour Bauchau, l'art est un itinéraire spirituel ancré dans l'existence et, de ce fait, il est avant tout une pratique. C'est un inestimable instrument de connaissance en ce qu'il est lui-même expérience incarnée au cœur de l'expérience existentielle. Mettant en jeu le corps, il éveille, par la sollicitation des sens, la mémoire enfouie, celle de la circonstance dans laquelle le processus de création puise secrètement sa nécessité et, réélaborant images et solutions, il restaure les énergies brisées et dévoile les potentialités annihilées. Il constitue une disposition de l'âme au changement et au dépassement de la souffrance, non seulement pour le créateur mais pour tous les êtres et c'est en cela même qu'il est spirituel. S'il ouvre sur un processus d'individuation, il ne se complaît pas dans la subjectivité dont il aide à se déprendre en conduisant le moi à "une instance plus vaste que celle qui a vécu l'expérience". S’il ne guérit pas toujours la souffrance, il l'empêche en tout cas de pétrifier l'être et déploie les conditions de sa transformation.

La création est aussi un combat titanesque qui oblige par nécessité à s'enfoncer très profondément dans l'inconnu de l'être pour y découvrir la source du processus de transformation. Le savoir-faire technique ne suffit pas. Le matériau résiste, une manière de dire que la conscience est impuissante à créer et qu'il est nécessaire de faire confiance à une autre instance, obscure celle-là, immaîtrisée, qu'il convient d'apprivoiser. Il doit y avoir disponibilité du créateur à sa vérité qui cherche à sourdre en marge de la rationalité. "Ce n'est ni une question de volonté, ni de bonne volonté. Poète n'est pas maître chez lui ", écrit Michaux cité par Bauchau dans L'Écriture à l'écoute. L'artiste doit ramener des tréfonds de la matière, dans un total effacement de son ego et une écoute absolue, "la circonstance" oubliée qui dicte sa nécessité et demande qu'on lui dessine une autre voie pour un autre avenir possible. Le Journal d'CEdipe sur la route témoigne à chaque instant de la difficulté à lâcher prise pour laisser advenir ce qui est enfoui et qui peine à émerger. Ce n'est pourtant qu'à cette condition que l'imaginaire se développe sur fond d'une totale disponibilité à l'inconscient. C'est ce que le poète formule dans L'Écriture à l'écoute: "L'imagination profonde est donc un rapport de liberté de notre part consciente avec cette merveilleuse et redoutable instance de la nuit ["la bouche d'ombre", disait Victor Hugo]. Pour connaître la liberté il

35 Outre la constellation d'écrits œedipiens, il conviendrait d'y associer L'Enfant bleu. 
faut d'abord n'avoir pas peur, devenir résolument soi-même et affronter les luttes inévitables car comme le dit CEdipe: la liberté douce n'existe pas. Ce rapport de liberté avec l'inconscient n'est pas un fait acquis, c'est un état où l'on parvient à travers un travail de toute la vie. On y accède par moments quand l'inconscient, se sentant écouté, apporte avec confiance à l'activité consciente ses matériaux, ses richesses, son étendue et aussi ses colères. Car sa colère veut dire espérance. ${ }^{36}$ Ainsi, comme la cure psychanalytique, la création artistique oblige à affronter la souffrance mais enclenche elle aussi un processus de transformation psychique qui redonne ses chances à la vie.

Henry Bauchau est bien un enfant de sa génération, la génération existentialiste qui a cherché, à travers bien des errements, des réponses aux traumatismes historiques auxquels elle a été confrontée. Pour cette génération, la souffrance ne peut être éludée et doit être nécessairement affrontée de plein fouet. Lart a de ce fait pour vocation de la méditer, de la creuser inlassablement dans l'espoir sans doute de s'en libérer. D'où la fascination de l'époque pour la psychanalyse qui elle aussi explore la souffrance existentielle. Participant de cette vision tragique de l'existence, les romans de Bauchau présentent l'art davantage comme une pratique introspective d'exploration et de libération de la souffrance que comme une finalité. C'est sans doute ce qui explique la rédaction des nombreux textes d'escorte que sont ses Journaux qui inlassablement interrogent le long et douloureux processus qui aboutit à l'œuvre en transformant l'être.

Bauchau semble distinguer plusieurs niveaux dans l'art, comme il en existe dans toute discipline spirituelle. La pratique qui, par le développement sensoriel qui lui est propre, réharmonise l'être, modifie et enrichit la perception, restaure la faculté relationnelle et rend possible la transformation psychique; la créativité qui aide à inventer des voies propres, des solutions singulières et à tisser des liens avec les autres; et l'œeuvre qui construit, au cours d'une expérience spirituelle inégalable - le Combat de Jacob avec l'Ange -, une connaissance supérieure, se constituant en vecteur de transmission de ce savoir auprès de la collectivité. Ainsi, Antigone ne prétend pas être une artiste quand elle sculpte et pétrit la matière, mais elle le fait pour trouver sa vérité et partager avec les autres; en revanche CEdipe, Alcyon et Io lorsqu'ils chantent, Clios lorsqu'il peint, sont des artistes accomplis qui sauront transmettre les modèles durables auxquels adhèrera la communauté. Ils possèdent une capacité exceptionnelle à partager les expériences existentielles qui donneront aux autres la force de chercher leurs propres solutions et d'inventer leur avenir.

36 Henry Bauchau, L'Écriture à l'écoute, op. cit., p. 149 
En somme, dans son ouvre littéraire, Bauchau pense l'art avant tout selon ses fonctions: thérapie psychologique, lien communautaire (au risque de la fusion), transmission, harmonisation spirituelle, outil de connaissance... Il ne semble pas y avoir chez lui de fonction esthétique autonome. Il ne se préoccupe pas tant de beauté que de vérité individuelle, de partage d'expérience et de maitrise de l'instinct par la forme, ce voile que l'artiste "jette sur le monstre inconscient" pour exercer une "action magique" sur lui "qui sans elle n'aurait d'autre souci que de se déployer sauvagement ${ }^{37}$. Lart a toujours chez lui une fonction initiatique et c'est à cette condition qu'il renouvelle la vie.

Certes Henry Bauchau a, par la littérature et la poésie mais aussi la peinture et la sculpture, fait œeuvre, sans se contenter d'un usage thérapeutique de l'art. Néanmoins, l'art joue chez lui un rôle particulier. Indissolublement lié à la transformation psychique dont il est l'adjuvant autant que la métaphore, il constitue le sujet même de son ouvre. Dans son travail d'artiste, processus créateur et transformation psychique sont ensemble - précisément parce qu'ils ont été indissociés dans sa propre existence - les garants du sens ${ }^{38}$.

Mais est-il possible d'approcher un tant soit peu ce sens déposé dans l'œuvre, en d'autres termes que ceux qu'a employés l'écrivain, dans "La circonstance éclatante ${ }^{39}$ par exemple qui met en avant la relation de rivalité avec son frère? L'hypothèse de cet article - mais elle reste à creuser et à vérifier preuves à l'appui - est que la réflexion sur l'art permet à Bauchau d'articuler deux thèmes philosophiques douloureusement ancrés dans son existence, avant, pendant et après la guerre: l'individu et la communautét ${ }^{40}$. Lart et l'analyse lui permettent, au cours de l'exil, d'envisager cette articulation autrement qu'à travers l'action sociale et politique qui caractérisait ses années de jeunesse. L’art va désormais constituer la réflexion en acte sur la spiritualité humaine, au-delà de tout ancrage religieux à proprement parler. Si la spiritualité consiste en la recherche du bonheur, non pas individuel mais partagé avec la communauté des hommes et de ce fait, en la délivrance de la souffrance, on peut dire que Bauchau adopte l'art comme l'indispensable compagnon de route sur ce chemin. Lart seul en effet parvient à établir

37 Ibid., pp. 149-150.

38 "On peut s'étonner de me voir parler autant de l'analyse. C'est que dans ma vie l'écriture et l'analyse se sont intimement liées. L'une a libéré l'autre et toutes deux ont continué à agir et à évoluer ensemble.", Ibid., p. 23.

39 "La Circonstance éclatante" est le premier chapitre de L'Écriture à l'écoute.

40 Tout d'abord proche des positions humanistes chrétiennes de Jacques Maritain et d'Emmanuel Mounier et membre des mouvements personnalistes, le groupe "Communauté" fondé par Henry Bauchau et Raymond De Becker en 1935 va susciter la défiance de ses anciens amis du fait de la confusion des idées exprimées par De Becker. "Communauté" deviendra de plus en plus sous sa plume un terme politique ambigu, évoquant plus la fusion dans un modèle à tendance totalitaire qu'un idéal spirituel comme il l'était à l'origine. 
une passerelle métaphorique entre l'expérience individuelle et l'universalité de l'expérience partagée: l'œuvre se détache de la circonstance qui l'a fait naître et de la quête personnelle du créateur pour devenir symbole de la condition humaine. L'artiste capte la souffrance d'un seul pour la partager avec tous et par cette catharsis l'alléger: CEdipe dépose ses statuettes sur le bord du chemin en offrande aux autres, Io partage son intuition unique d'Antigone avec les êtres qui l'écoutent, Edipe transmet la mémoire du monde par son chant... Par l'attitude esthétique, chaque être humain soulage à son tour l'artiste de sa souffrance et progresse sur le chemin de la connaissance. Par le travail artistique, l'homme construit son humanité : il affronte tout d'abord en toute lucidité les exigences du processus d'individuation qui l'empêcheront de céder aux sirènes fusionnelles puis s'élève à un autre niveau qui est celui de la condition humaine partagée. Le face à face de Jacob et de l'Ange ne conduit nullement à l'abolition de l'homme dans la divinité, mais à la pacification intérieure du guerrier dont l'humanité triomphe, au dépôt de ses armes et à sa transformation en guide spirituel et transmetteur de la vie.

Joëlle Caullier

Université de Lille III-Nord de France 